\title{
BREAKING WAVE KINEMATICS, LOCAL PRESSURES, AND FORCES ON A TRIPOD SUPPORT STRUCTURE
}

\begin{abstract}
Arndt Hildebrandt ${ }^{1}$ and Torsten Schlurmann ${ }^{2}$
This paper presents breaking wave loads on a tripod structure from physical model tests and numerical simulations. The large scale model tests $(1: 12)$ are described as well as the validation of the three dimensional numerical model by comparison of CFD wave gauge data and pressures with measurements in the large wave flume inside and outside the impact area. Subsequently, the impact areas due to a broken wave, a curled wave front as well as for wave breaking directly at the structure with a partly vertical wave front are compared to each other. Line forces in terms of slamming coefficients with variation in time and space are derived from CFD results and the velocity distribution is presented at the onset of wave breaking. Finally, the results are briefly discussed in comparison to other slamming studies.
\end{abstract}

Keywords: Slamming coefficient; Wave impact; Line force; Wave kinematics; CFD; Impact simulation

\section{INTRODUCTION}

The motivation for this study is driven by the growing offshore market with thousands of projected wind energy converters within the next years. In 2010, wind energy increased the most in the field of renewable energy, followed by hydropower and photovoltaics (Global Status Report - REN21, 2011). This underlines the global request for wind energy and the currently growing demand for offshore wind solutions. Detailed understanding of the extreme, dynamic wave loads on offshore structures is essential for an efficient design and mass production. The impact on structures is a complex process and further studies are required for more detailed load assessments, which is why breaking wave loads were investigated by the research project "GIGAWIND alpha ventus - Subproject 1" (see acknowledgement) within the network "Research at Alpha VEntus" (RAVE).

The above-named aspects provide the motivation to further increase knowledge about the hydrodynamics of the flow field, pressure characteristics, and total forces due to impacting waves on offshore foundation structures. Wienke \& Oumeraci (2005) provide a certified approach based on theoretical formulations including an empirical curling factor. Assumptions about the wave profile and wave celerity are necessary to calculate the time dependent line forces, which are constantly distributed along the cylinder's span for each point in time. Results from laboratory tests and numerical simulations within the above named research project suggest that the idealized theory as well as the underlying assumptions according to Wienke \& Oumeraci (2005) might overestimate slamming loads. Generally, designing with conservative loads is recommended practice and reasonable for naval architecture and offshore structures with human loss or dramatic consequences for the environment involved in case of failure. However, for the targeted mass production of offshore wind energy converters (OWEC) more details on impact loads support the balance of risk analysis and cost effectiveness.

Large scale laboratory tests (1:12) with breaking waves have been carried out at the Large Wave Flume of the "Forschungszentrum Küste" (FZK, Hanover) to reveal more detailed insights on the region of impact, development and duration. This paper describes results on breaking wave characteristics and detailed loads based on physical model tests and numerical simulations. Temporally and spatially distributed slamming loads are presented for different stages of wave breaking ahead of the tripod foundation structure.

\section{PHYSICAL MODEL}

Figure 1 illustrates the cross-section of the large scale experiments (1:12) with the tripod model in the large wave flume ("Großer Wellenkanal" - GWK), at the coastal research center in Hanover, Germany. The wave flume is $7 \mathrm{~m}$ deep, $5 \mathrm{~m}$ wide and $330 \mathrm{~m}$ long. Due to parallel and subsequent model tests on scour development around the tripod structure (Stahlmann and Schlurmann (2012), a trapezoid sand profile is integrated in the test setup. After $70 \mathrm{~m}$ with a water depth of $3.7 \mathrm{~m}$ the sand profile increases along $24 \mathrm{~m}$ to a height of $1.2 \mathrm{~m}$, which corresponds to a slope of 1:20. Therefore, the water depth is $2.5 \mathrm{~m}$ above the $34 \mathrm{~m}$ long horizontal section until the slope on the rear side decreases with 1:20 up to $152 \mathrm{~m}$ behind the zero position of the wave maker. 24 wave gauges with $0.7 \mathrm{~m}$ distance to the southern channel wall are installed along the wave flume to capture the water surface elevation and the development of the breaking waves, which is why the horizontal spacing of the wave gauges

\footnotetext{
${ }^{1}$ Franzius-Institute for Hydraulic, Waterways and Coastal Engineering, Leibniz Universität Hannover, Nienburger Straße 4, 30167 Hannover, Lower Saxony, Germany, Research staff

${ }^{2}$ Franzius-Institute for Hydraulic, Waterways and Coastal Engineering, Managing Director \& Chair
} 


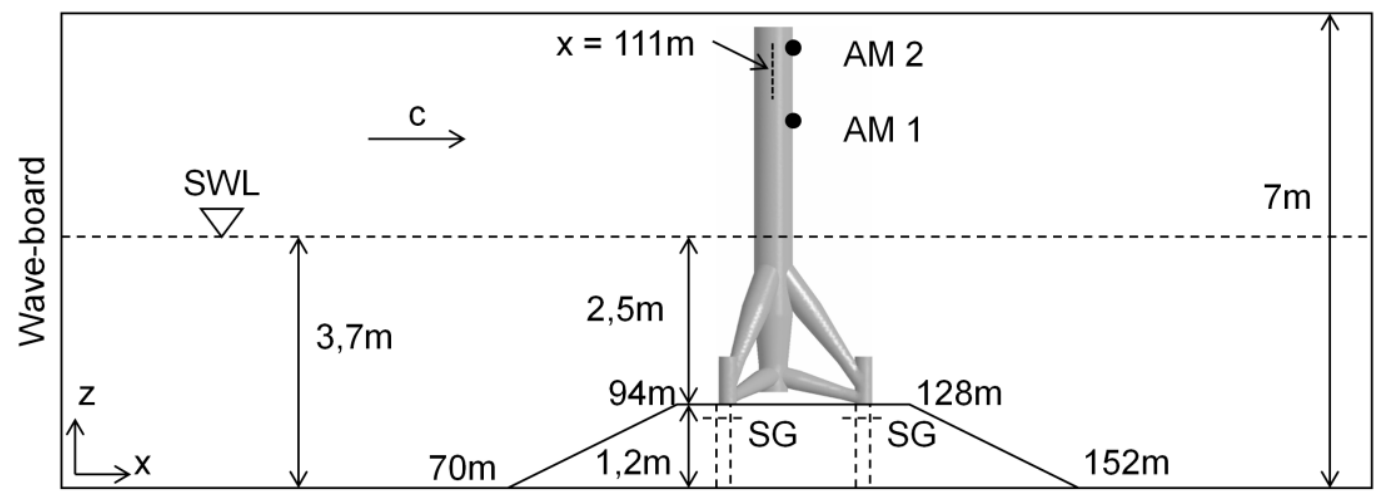

Figure 1. Cross-section of the experimental setup with still water level (SWL), acceleration meter (AM), and strain gauges (SG).

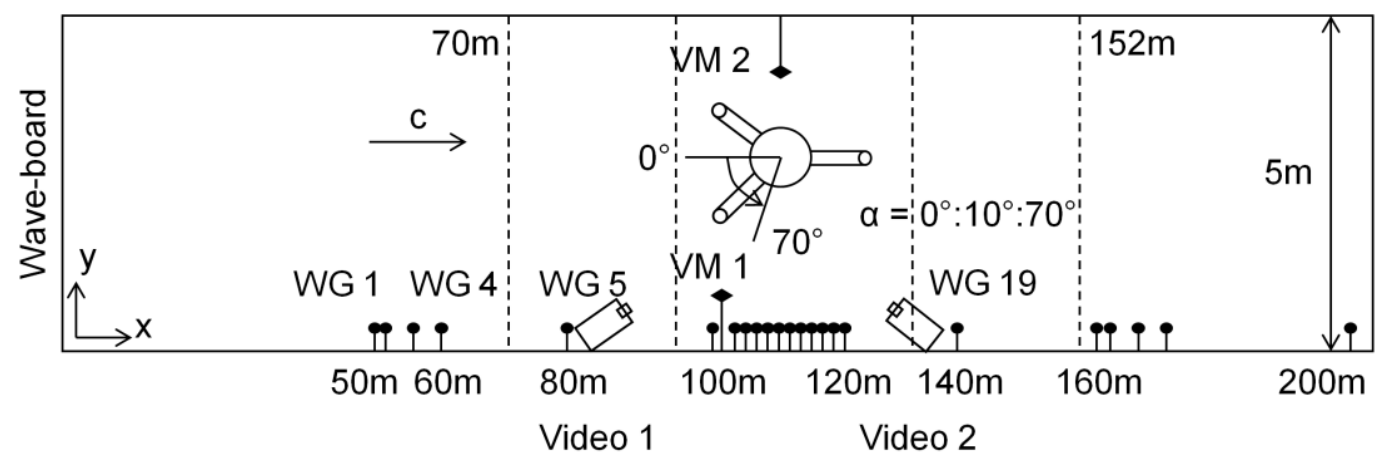

Figure 2. Plane-view of the experimental setup with wave gauges (WG), velocity meters (VM), Video cameras, and angle of rotation.
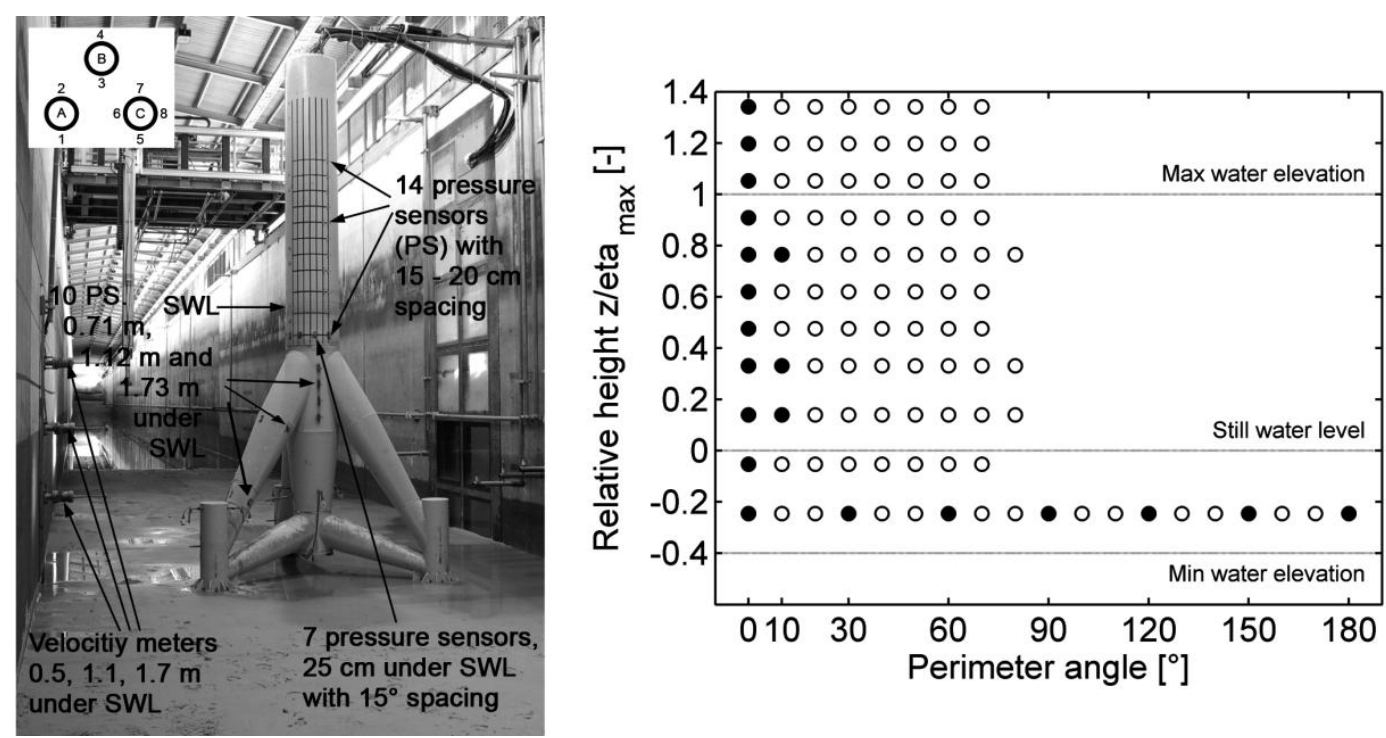

Figure 3. Left, large scale tripod structure (1:12) with water pressure sensors (PS), strain gauges (SG), acceleration meters (AM), and velocity meters on a level with the tripod main column. Right, positions of water pressure sensors in the rotating section of the tripod main column. 
becomes smaller in the near field of the tripod and partly reduces down to $0.25 \mathrm{~m}$ (Figure 2). Furthermore, six electromagnetic velocity meters are installed to measure the horizontal (x-direction) and vertical (z-direction) water velocities in a two-dimensional plane parallel to the channel wall. Three current meters are located $101 \mathrm{~m}$ behind the wave maker $(6 \mathrm{~m}$ behind the end of the increasing slope) with $0.6 \mathrm{~m}$ distance to the Southern wall. The other three current meters are positioned on a level with the main column of the tripod at $111 \mathrm{~m}$ with $0.4 \mathrm{~m}$ spacing to the northern wall of the flume (Figure 2). At both locations the velocity meters are positioned $0.5,1.1$, and $1.7 \mathrm{~m}$ below the still water level (Figure 3, left) and are submerged in the water at all times during the experiments. Two video cameras are installed at the southern wall of the wave flume (Figure 2) to capture the wave impact with 200 frames per second. They are mounted in front of, next to, behind, and above the main column to record the repeated breaker types from different viewpoints.

The main column of the tripod has a diameter of $0.5 \mathrm{~m}$ with an upper rotating section and 20 installed pressure sensors. Figure 3 (right) illustrates the locations of the 20 instrumented pressure sensors for the $0^{\circ}$ angle position by the filled circles. The upper section of the tripod is shifted from $0^{\circ}$ up to $70^{\circ}$ angles by $10^{\circ}$ intervals. A wide range of measuring positions along the cylinder's span as well as along the perimeter is covered in this way, which results in the plotted grid shown in Figure 3. Ten additional pressure sensors are instrumented in the stationary lower part of the structure. Three sensors record the pressures approximately $1.73 \mathrm{~m}$ below the still water level (SWL) at the upper brace A according to Figure 3. Likewise, three other sensors are installed $1.12 \mathrm{~m}$ below the SWL and the remaining four pressure sensors are vertically aligned at the upper part of the main column, $0.56,0.71$, 0.86 , and $1.01 \mathrm{~m}$ below SWL. In addition to the pressure sensors, two three-dimensional acceleration meters are installed in the main column $1.18 \mathrm{~m}$ and $2 \mathrm{~m}$ above SWL, which recognize the onset and intensity of the tripod's movement. The tripod is fixed on a steel pipe substructure, which is submerged in the sand and connects the three legs of the tripod with the bottom of the wave flume. Eight strain gauges are positioned at the three steel pipes A, B, and C as sketched in Figure 3 (left). They are used to estimate time dependent total loads on the tripod structure due to their position right below the bolted joint connection of the tripod and the substructure.

Wave gauges, velocity meters, and the wave board are not subject to rapid changes in the time series and were sampled with $100 \mathrm{~Hz}$. This is not the case for the acceleration meters and especially not for the pressure sensors in the slamming area. Therefore, the strain gauges and acceleration meters were sampled with $600 \mathrm{~Hz}$ and the pressure sensors with $10 \mathrm{kHz}$.
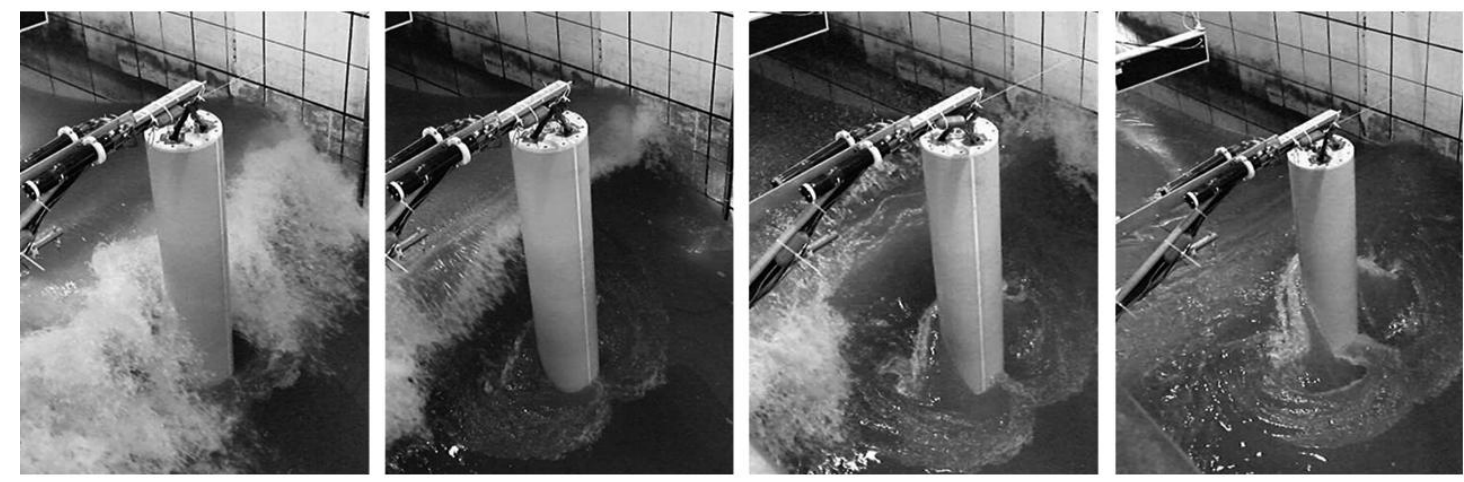

Figure 4. Snapshots of breaking wave types 1 to 4 at the tripod main column from left to right; starting with the impact of the broken wave, curled wave front, partly vertical wave front, and the steep non-breaking wave just before the onset of wave breaking.

\section{Test Program}

Four different wave types with various breaking distances to the main column of the tripod are tested in the experiments. Figure 4 shows snapshots of the four cases 1-4 from left to the right. All breaking waves are generated by focusing wave packets with a characteristic wave height of $0.8 \mathrm{~m}$ and a peak period of 4 seconds. The characteristic wave parameters are kept constant for all test cases and only the focusing point of the wave packet is shifted for the load cases 1-4.

For load case 1, wave breaking sets in $6 \mathrm{~m}$ before the tripod main column. The wave is a broken wave with a foamy wave front due to the mixture of entrapped air and the mass of water, similar to 
stage V described by Chan et al. (1995). Load case 2 is generated with a wave breaking position starting $4 \mathrm{~m}$ in front of the main column. The wave has a concave wave front with jet formation or curling breaker tongue, which hits the cylinder at higher elevations before falling downward into the water; similar to Stage III+IV according to Chan et al. (1995). Load case 3 starts breaking right in front of the tripod main column with a partly vertical wave front at the crest and corresponds to stage II by Chan et al. (1995). Load case 4 is becoming instable at the main column and thus breaking behind the structure. This represents the quasi-static load component without slamming loads, however, with a non-breaking wave shape at the cylinder as similar as possible to the combined wave loads.

\section{Synchronization and reproduction of tests}

In order to measure the spatial and temporal distribution of the wave impact the central cylinder is rotated along the vertical axis in $10^{\circ}$ steps. The rotational section provides a relatively high resolution of measuring points using a limited number of pressure sensors. Prerequisite for this approach is a good reproducibility of the breaking wave forms as the individual experiments are plotted and analyzed in one combined grid. The movement of the wave maker in conjunction with the constant water level is one parameter to cross-check the wave shapes as well as all measurements at the structure. Furthermore, wave gauge records before, after, and at the onset of wave breaking are analyzed to assess the reproduction of the equally generated waves. Generally, the wave gauges are in good correlation and the standard deviation is small for locations up to the occurrence of broken wave fronts. Entrapped air and the turbulent state of the propagating wave front are challenging for the evaluation of reproduced waves at locations after wave breaking.

The problem of wave gauge records influenced by air entrainment is excluded for the time series of lower positioned hydrodynamic pressure measurements at the tripod. Figure 5 (left) shows two sets of pressure signals with a $300 \mathrm{~ms}$ shift for better illustration purposes. Each set represents three experiments with identically generated waves, but with different angle positions of the rotational tripod section. The sensors are located at the relative height 0.14 according to the grid in Figure 3. Likewise, Figure 5 (right) illustrates two sets of pressure records at a relative height of 0.33 . The similar time series of the pressure signals indicate the good reproducibility of the breaking waves directly at the structure. The exemplarily plotted signals are taken from load case two with a moderate amount of air entrainment at the crest. Rise time, the peak shape as well as the distinctive drop seen at $72.3 \mathrm{~s}$ in Figure 5 (left \& right) are reproduced by each test wave. Furthermore, the standard deviations of the pressure signals are very small even around the peak.
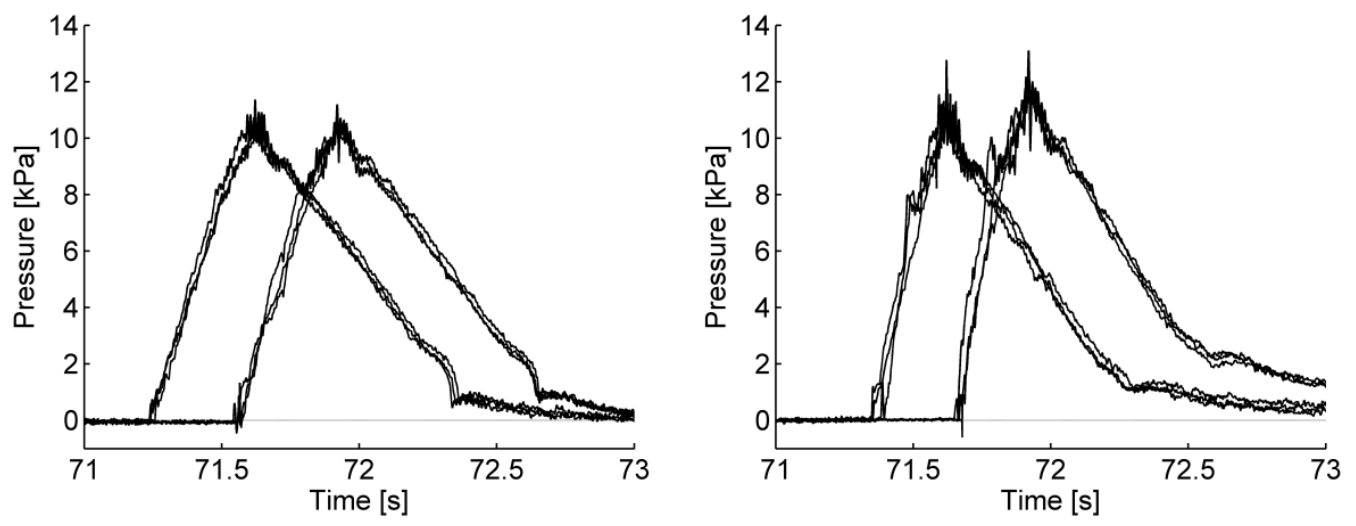

Figure 5. Similarity of hydrodynamic pressures at relative heights $z /$ eta $a_{\max }=0.14$ (left) and 0.33 (right) for various test waves.

In addition to the evaluation of the reproducibility the well correlated signals during rise time and the peak characteristics are especially useful for the synchronization of the tests. Therefore the horizontally and next to each other arranged pressure sensors described in the prior paragraph are used to couple the experiments. Since the time series are in good agreement the data sets with angle positions between $0^{\circ}$ and $70^{\circ}$ are synchronized by the rise time and peaks of the overlapping sensor positions of the rotating section. For this purpose sensors at positions above the still water level are favorable compared to the pressure sensors located at the stationary part of the tripod below SWL. As the wave front shape is the most important part of a breaking wave the synchronization of the front should be as accurate as possible in the order of milliseconds. Pressure sensors below SWL do not have 
a pointed peak, but a curved crest instead. In contrast to that, the pressure signals plotted in Figure 5 have a specific onset of the rise time, are close to the impact zone, and have a pointed peak. This provides a far more accurate synchronization, better reconstruction and correlation of the wave front. Theoretically, the two pressure sensors at the higher position z/eta $\max _{\max }=0.76$ (Figure 3) offer even more accurate correlations, since they are positioned inside the impact area. Practically, this location is strongly affected by air bubbles in the wave crest and by additional oscillations of the structure. Therefore time series at that location show considerable variations and small random peaks, which are difficult to correlate. Synchronization of the pressure signals exclusively on the basis of the wave gauges leads to inaccurate results for the short-timed impulsive pressures. Firstly, this is due to the air entrainment and slight changes of the wave front, which are barely distinguishable in wave gauge records. Secondly, the lateral distance between the wave gauges and the main column is nearly $2 \mathrm{~m}$ and wave breaking is not ideally two-dimensional across the flume width, as can be seen in Figure 4. This includes additional uncertainties for the synchronization based on wave gauges, since the wave tongue might be different at the wave gauge and the main column. Therefore, the wave gauges as well as the pressure sensors located below the SWL are only used for plausibility checks and the synchronization is performed with the pressure sensors at the relative heights 0.14 and 0.33 above the SWL. Subsequently, the measuring positions are combined to one grid for the investigation of the spatial and time dependent pressure development around the main column as well as to validate the numerical model.
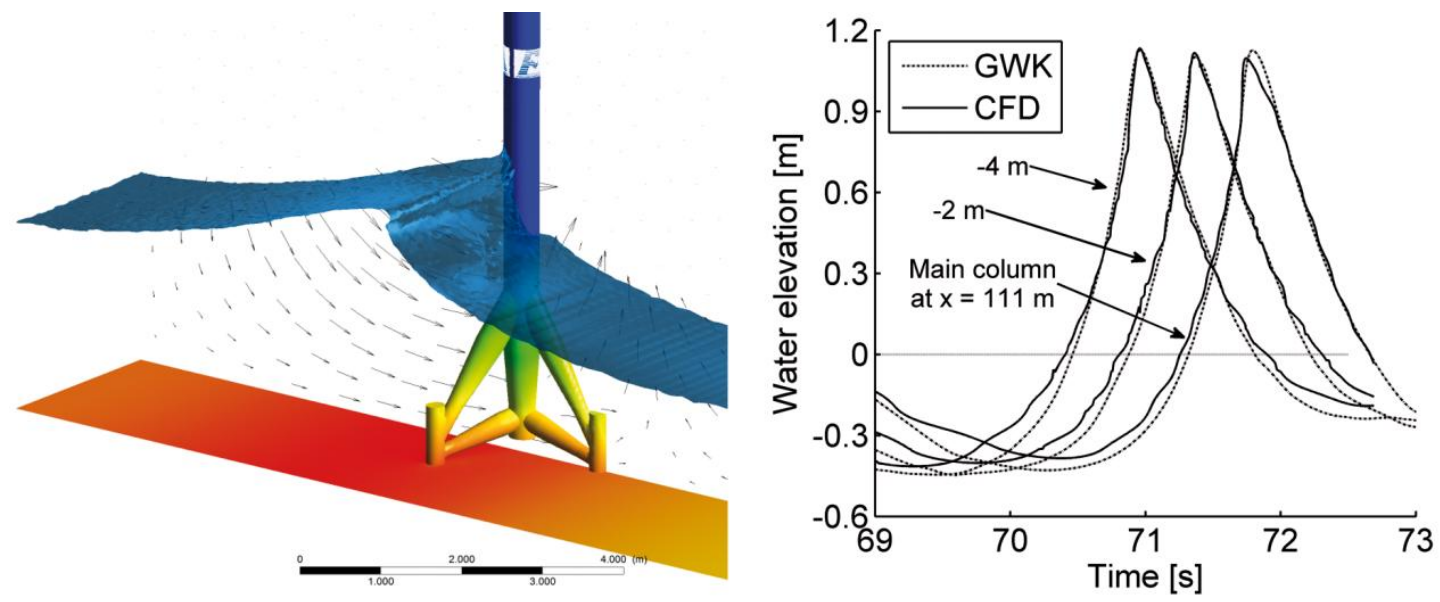

Figure 6. Wave breaking and impact on the tripod structure in the three dimensional CFD model (left) as well as wave gauge records of the developing breaking wave in the large wave flume and in the numerical model at three positions near the cylinder

\section{NUMERICAL MODEL}

Numerical modeling is connected with physical models e.g. to set boundary conditions and for validation. However, validated numerical models provide several advantages in comparison to physical models. Deterministic breaking waves are reproduced exactly so that the position of the structure in regard to the impact can be modified without affecting the wave geometry, which is not always possible in large scale experiments. In general, parameters such as hydrodynamic pressure or velocity meters are available for the complete flow domain under the wave and around the structure, whereas in laboratory experiments data points are taken at individual positions and the equipment can even interfere with the flow. The three-dimensional flow simulations described here were simulated with the software package Ansys CFX ® on the basis of the volume of fluid method.

At first the development of the wave from the wave maker until shortly in front of the structure was simulated in a quasi-two dimensional model containing only one element in the plain of projection. The velocities immediately before the onset of wave breaking were exported and implemented as boundary conditions in the 3D model, which is illustrated on the left in Figure 6. The simulation time of a wave impact in the 3D model strongly depends on the flow history of the wave. It either starts at the time point described above or can be shortened significantly by using the flow field of a wave that has already been simulated so that only a short period of time immediately before the impact and the wave impact itself have to be simulated in the 3D model. While the first approach takes about a week 
with 24 CPUs to finish the second one can be calculated within $48 \mathrm{~h}$. Due to large velocity gradients during wave impact, small time steps are required in order to keep the residues and numerical stability.

\section{Validation of the Numerical Model}

The setup of the numerical model is validated by comparison of water level and pressure data with results from the GWK experiments. Out of the first three physically tested breaking waves load case two provides the best reproducibility with relatively low standard deviations of the averaged pressure time series in the region of wave impact. All load cases show small standard deviations for the pressure signals outside the impact area as well as for the wave gauges. However, load case one is characterized by strong pressure fluctuations near the impact area due to the highly turbulent mixture of air and water. In contrast to that load case three is defined by a sharp wave profile with almost no air entrapped. Even though, marginal variations of the vertical wave profile generate strong pressure deviations near the wave crest and complicate the local comparison of pressure measurements to the numerical model. Therefore, load case two is presented in the following in combination with the same numerically simulated load case. Comparisons to all load cases including statistical values of wave gauges, velocity meters, as well as pressures around and inside the corresponding impact areas will be published soon in the framework of the author's dissertation.

The plot in Figure 6 illustrates the records of three wave gauges of load case two positioned at the main column of the tripod as well as $2 \mathrm{~m}$ and $4 \mathrm{~m}$ in front of it. The data shows the water elevation over time whereby the solid lines represent the numerical model and the dashed lines experimental data from the large wave flume. The maximum water elevation slightly decreases with decreasing distance to the tripod because of the curling and thus, downward falling, wave crest. Principally, all three time series are in good correlation and the wave gauges of the simulation reproduce the measured results with sufficient accuracy. Minor differences are detectable between trough and crest of the $1.46 \mathrm{~m}$ high wave, which results in a slightly reduced wave steepness of the simulated wave. This kind of deviation is on the one hand caused by small variations of the breaking wave front in the laboratory. On the other hand the air that is included in the wave crest affects the wave gauge records at the wall of the large wave flume.
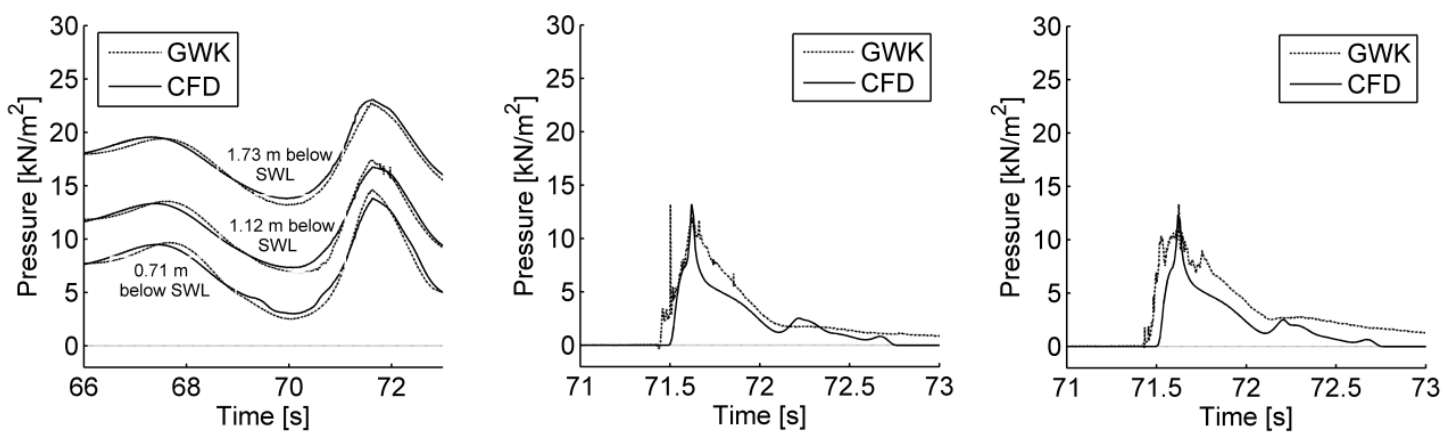

Figure 7. Time series comparison of hydrodynamic pressures in the substructure of the tripod (left, and indicated in Figure 3 by arrows) as well as in the relative height $z / e t a_{\max }=0.62$ at the perimeter angle $0^{\circ}$ (middle) and $20^{\circ}$ (right). Solid lines show data from the CFD model and dashed lines GWK measurements.

In addition to the wave gauge data the left plot in Figure 7 compares three time series of the hydrodynamic pressures measured at the tripod. The positions of the sensors are located $0.71 \mathrm{~m}, 1.12 \mathrm{~m}$ and $1.73 \mathrm{~m}$ below the mean sea level and indicated by the arrows in Figure 3 (left). Due to the highest hydrostatic pressure the top time series represents the lowest pressure sensor and vice versa. In contrast to the wave gauge records, these results are not affected by air bubbles. Again, the simulations agree well with the test data, e.g. the wave profile fitting with steepness, minimum and maximum values over the whole wave period. However, the pressure sensors beneath the water column do not accurately detect the slight but significant changes in the shape of the wave tongue so that additional pressure sensors at higher elevations are used to compare further details from experiments and simulations.

Two exemplary plots show the pressure time series in a relative height $\mathrm{z}^{\prime}$ eta $\mathrm{max}_{\max }=0.62$ in the middle and on the right in Figure 7, which is located shortly under the impact area. Analogous to the comparisons above the solid lines illustrate the numerical calculations while the dashed lines show averaged pressure signals of five test cases with the main column in zero degree orientation (middle plot) and of four equally generated test waves with 20 degrees orientation on the right. Basically, both 
orientations show good agreements of simulated and tested pressure data, only with a small time shift after the peak value. The numerical results match the maximum experimental pressures of nearly 15 $\mathrm{kN} / \mathrm{m}^{2}$ as well as the rising times. Small differences between the time series are observed with regard to high frequent fluctuations, which are only present in the experiments due to the entrapped air in the wave crest. As mentioned above these fluctuations are of minor importance in this load case and have more influence on the pressure records of the broken wave. The pressures under the wave crest last a bit longer in the physical model than observed in the numerical model. In addition to numerical reasons the signals also deviate due to variations of the bow wave in both models, which occurs on the front side of the cylinder while the water level is rapidly rising up during the steep approaching wave front. As previously observed by the wave gauge records in Figure 6 (right) and by the pressure measurements below SWL in Figure 7 (left) the full wave period and pressure amplitudes of the physically and numerically modeled breaking waves are practically similar. The additional comparison of local pressures at higher levels underlines the validity of the numerical model up to the lower boundary of the impact area. The following section with details on the developing pressures in the impact area will illustrate the reproducibility of the breaking wave by the numerical model as well, even though differences are present. Additionally, the time dependent total forces are in good correlation, too, which indicates the validity of the integrated pressures on the whole numerical tripod structure. This is beyond the scope of this paper about local slamming forces and will be published in the former mentioned dissertation.

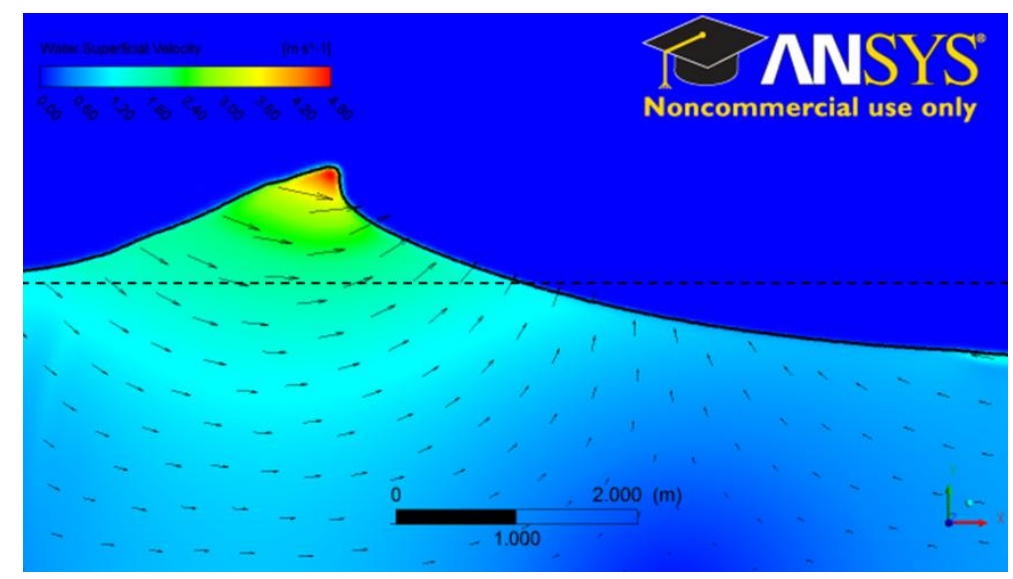

Figure 8. Water velocities under the breaking wave just before wave breaking.

\section{WAVE KINEMATICS, LOCAL IMPACT PRESSURES AND FORCES}

\section{Wave Kinematics}

Fluid velocities are of major interest for force calculations, since impact loads are proportional to the squared fluid particle velocity hitting a structure's surface. Furthermore, breaking waves have the highest water velocities at the surface of all waves and the stagnation pressure based on a constant flow velocity is commonly used in literature to normalize pressure data. With regard to the slamming problem the phase velocity of the breaking wave is a very useful reference velocity, since higher velocities indicate the region of wave breaking and probable locations of impact loads.

The phase velocity of the focusing wave packet in the large wave flume is estimated from wave gauge recordings and video analysis in the vicinity of the tripod. Not the velocity of the whole wave but the water velocity of the wave crest just before wave breaking is of special interest in regard to the slamming problem, since this water mass contributes the impulsive forces. The video observations as well as the analyzed wave gauge records up to $10.75 \mathrm{~m}$ in front of the main column lead to a representative crest velocity during the impact of $4.8 \mathrm{~m} / \mathrm{s}$. This value agrees with observations of the crest velocities in the CFD model. Since the wave gauge records and the wave shape of both models correlate well to each other the time resolved wave kinematics of the breaker are available in the CFD model. Figure 8 shows the wave kinematics of the breaking wave at the onset of breaking shortly before the wave crest starts to curl over for a cross-section next to the tripod and not influenced by the structure. The dashed line illustrates the still water level while the arrows represent vectors of the flow field combined with the colored magnitudes. As anticipated from wave theory, horizontal water 
velocities are observed under the wave crest. The velocity distribution from the wave crest down to the bottom changes rapidly above the still water level and remains relatively constant further down below. Maximum water velocities from 4.2 to $4.8 \mathrm{~m} / \mathrm{s}$ cover the upper $25 \%$ of the positive maximum water

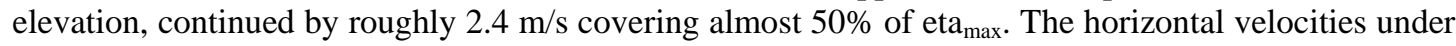
the wave crest and below the still water level range from 1 to $2 \mathrm{~m} / \mathrm{s}$ with small velocity gradients. The velocity distribution shows that only less than $25 \%$ of the upper wave crest contains sufficiently high velocities to overcome the phase speed of the wave and to curl over. Hence, the developing breaker tongue is relatively thin and the observed breaker type is an intermediate case of spilling and plunging breaking. This type of wave breaking was tested within the framework of the research project "Gigawind alpha ventus" (see acknowledgement) for offshore wind farm locations near the research platform FINO I. The water depth around FINO I is about $30 \mathrm{~m}$ and extreme wave breaking is assumed to be rather spilling type or moderate plunging breaking for that location, which is adopted for this study and the further descriptions in the following.
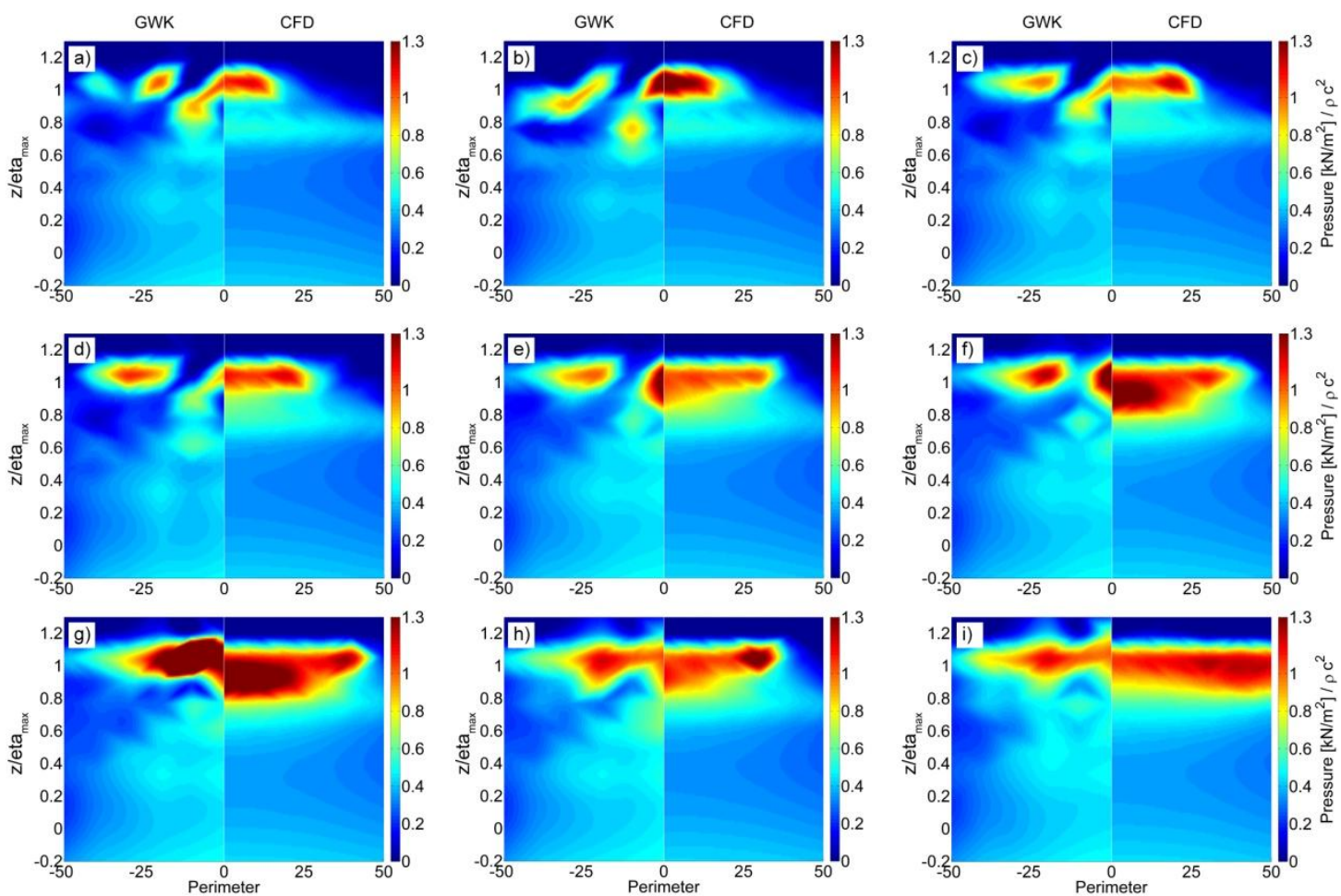

Figure 9. Comparison of normalized impact pressures from large wave flume experiments (GWK) and from numerical simulation (CFD) at relative time steps $t / T_{i}=t /(R / c): \quad 0.01$ (a), 0.03 (b), 0.05 (c), 0.10 (d), 0.15 (e), 0.20 (f), $0.25(\mathrm{~g}), 0.35(\mathrm{~h}), 0.45$ (i).

\section{Local Impact Pressures}

Figure 9 shows plots of the pressure distribution at the main column of the tripod structure. Slamming loads are symmetric to the span of the cylinder at the perimeter angle zero, which is why the results from the large wave flume (GWK) experiments are shown on the left side in direct comparison to the CFD results on the right side for each subplot. The abscissa shows the perimeter angle of the cylinder up to 50 degrees to both sides and the ordinate represents the vertical relative height " $\mathrm{z} / \mathrm{eta} \mathrm{max}_{\max }$. Last mentioned is defined by the vertical coordinate " $z$ ", counting zero at the still water level, divided by the maximum water elevation "eta $\max$ ". Pressures are referenced to the stagnation pressure, which is water density $\left(\rho=1000 \mathrm{~kg} / \mathrm{m}^{3}\right)$ multiplied by the squared wave celerity $(c=4.8 \mathrm{~m} / \mathrm{s})$. The time shifts between the plots are referenced to the time of immersion " $\mathrm{T}_{\mathrm{i}}$ " defined by the duration the wave crest takes to submerge the cylinder's front half. This is calculated by the phase velocity of the wave "c" divided by the radius " $\mathrm{R}$ " of the cylinder. The first contact of the wave tongue with the cylinder front sets the point of time $t / T_{i}=0$, while $t / T_{i}=1$ refers to the submerged cylinder front up to the center line by the wave tongue.

Plot a) in Figure 9 shows the pressure distribution around the main column of the tripod shortly after the wave tongue has encountered the cylinder $\left(t / T_{i}=0.01\right)$. The tongue of the slamming wave hits 
the cylinder at the level of the wave crest $\left(z /\right.$ eta $\left._{\max }=1\right)$ in both models and the pressure is roughly $1 \rho \mathrm{c}^{2}$ at the cylinder's span. Subsequently, the pressure spreads horizontal up to 30 degrees across the perimeter in the CFD model within $10 \%$ of $\mathrm{T}_{\mathrm{i}}$ (plot d). The impact area nearly remains constant in vertical direction during that time and values higher than $0.8 \rho c^{2}$ only occur between $z /$ eta $a_{\max }=0.9$ and 1.1. This initial and horizontal spreading is connected to the immersion of the hitting wave tongue. However, the wave shape in the physical experiment is not strictly two dimensional as can be seen in the snapshot of load case two in Figure 4. The wave breaking starts at the channel walls and propagates to the middle of the flume. Furthermore, the wave tongue also consists of single droplets and entrapped air in contrast to the more idealized tip of the wave tongue in the CFD model. This is why the impact of the more naturally breaking wave in the physical model simultaneously starts at various positions between $0^{\circ}$ and $30^{\circ}$ (plots a-d). Nevertheless, the pressure spots in the experiments and the CFD results show comparable intensity. Between 0.15 and $0.25 \mathrm{~T}_{\mathrm{i}}$ the pressure becomes more intensive and spreads from $0^{\circ}$ up to $25^{\circ}$ in both models (subplots e - g). Pressures up to $1.5 \rho c^{2}$ are reached in the CFD model for $\mathrm{t} / \mathrm{T}_{\mathrm{i}}=0.2$ (plot $\mathrm{f}$ ) in comparison to a maximum value of $1.7 \rho \mathrm{c}^{2}$ in the physical model tests. Higher differences are observed for $\mathrm{t} / \mathrm{T}_{\mathrm{i}}=0.25$ (plot $\mathrm{g}$ ) with maximum CFD pressures of $1.7 \rho \mathrm{c}^{2}$ in contrast to $3.5 \mathrm{\rho c}^{2}$ recorded in the laboratory tests. Further comparison of the impact area at various time steps shows that the intensive pressure field in the CFD simulation generally exceeds the area covered by high pressures in the experiments. Impact pressures obtained by the CFD simulation and values greater

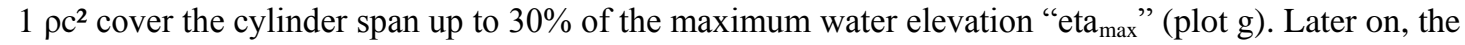
impact region becomes narrower again at high perimeter angles and extends approximately along $20 \%$ of eta $a_{\max }$ with reduced pressures around $1 \mathrm{cc}^{2}$ (plots $\mathrm{h}, \mathrm{i}$ ). The impact pressures observed by the laboratory tests stretch over $30 \%$ of eta $\max _{\max }$ along the cylinder front as well, however, do not occur at higher angles than 30 or 40 degrees (plot g-i). As already seen at the initial stages of the wave impact, the measured pressures at later stages with $\mathrm{t}>0.35 \mathrm{~T}_{\mathrm{i}}$ show fluctuations as well, although less dominant as before (plots h, i).

With regard to the validation of the numerical model, the highest differences between the physical and numerical results are found in the upper section $\left(z /\right.$ et $\left.a_{\max }>0.62\right)$ of the main column in the vicinity of the hitting wave tongue. On the one hand the models differ by the outer extension of the impact area and by the under prediction of the local maximum value at $t / T_{i}=0.25$ in the CFD model. On the other side the time dependent spreading of the impact in combination with the vertical positions along the cylinder's span, as well as the pressure intensities are in good agreement and show the capability of the numerical model. Beside the pressure and wave gauge data presented in this paper, velocities as well as total forces on the tripod structure are recorded in the experiments. Last mentioned are in good and even better correlation to the numerical model as the pressure in Figure 9, since the total force measurements scatter significantly less than the pressure recordings above $z /$ eta $a_{\max }>0.62$. The reason is found in the partial contribution of the impact pressure to the total force, which is composed of the impact force and the considerable amount of the quasi-static load. The numerical model offers detailed analysis of the highly time and space dependent impact forces described in the next section.

\section{Local Impact Forces}

Figure 10 to Figure 12 show temporally and spatially resolved slamming coefficients for the three illustrated types of wave breaking, which correspond to the snapshots 1-3 in Figure 4. Abscissas show the relative time normalized by the previously described time of immersion $(\mathrm{R} / \mathrm{c})$ and the ordinates show the relative height $\mathrm{z}^{\mathrm{e}} \mathrm{ta}_{\max }$ analogous to the pressure plots in Figure 9, ranging from zero at the still water level up to 1.6 times the position of the wave crest. Each point shows a slamming coefficient for a specific point in time defined by the local line force at the cylinder span divided by the line force commonly used in literature based on the stagnation pressure $\left(\rho \mathrm{Rc}^{2}\right)$ with fluid density $\rho$. The line forces are calculated by the integrated pressures of the CFD model along the full circumference of the tripod's main column, while the constant reference value is the flow force induced by a steady flow with velocity $\mathrm{c}=4.8 \mathrm{~m} / \mathrm{s}$ acting on the diameter of the main column $(2 * \mathrm{R})$.

Furthermore, the solid white lines give the water level elevations on the front and rear side at the main column. Thereby the upper line gives the time dependent location of the bow wave before the impact and the wave run-up when the tongue encounters the cylinder. The difference between the upper and lower solid white lines shows the section along the cylinder with almost no water pressure acting 

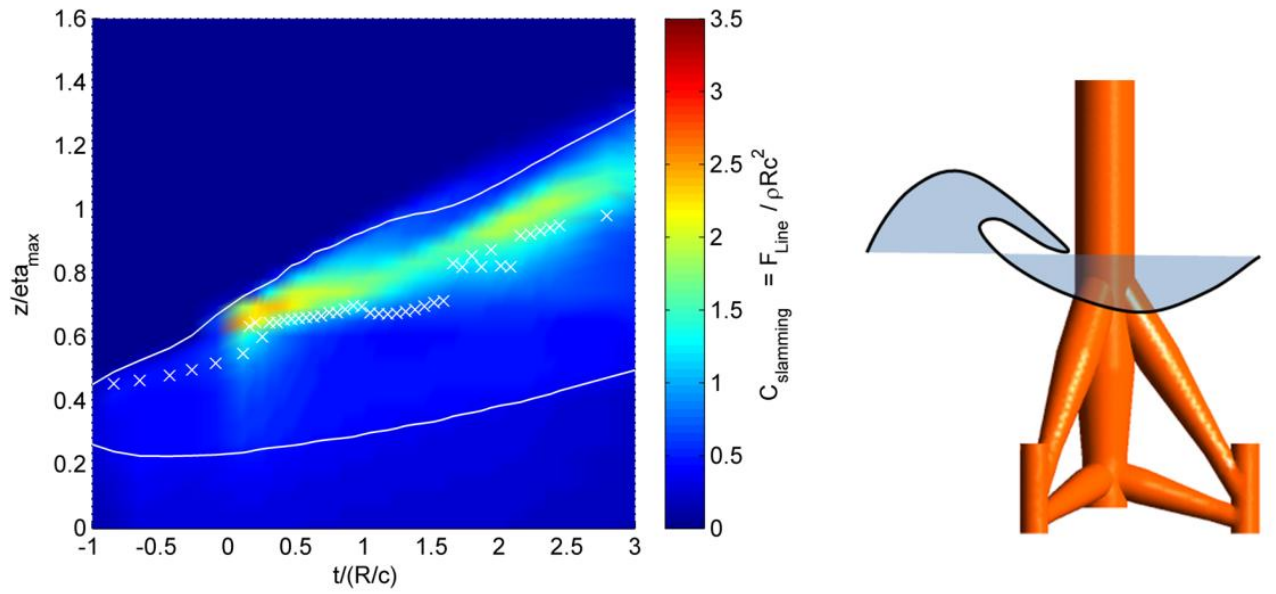

Figure 10. Slamming coefficients of LC 1 over time normalized by time of immergence (radius/wave velocity) and versus relative height " $z /$ eta $_{\max }$ " with water elevations at the cylinder's front (upper solid line) and rear side (lower solid line); the x-marker line shows the wave gauge unaffected by the structure for orientation.
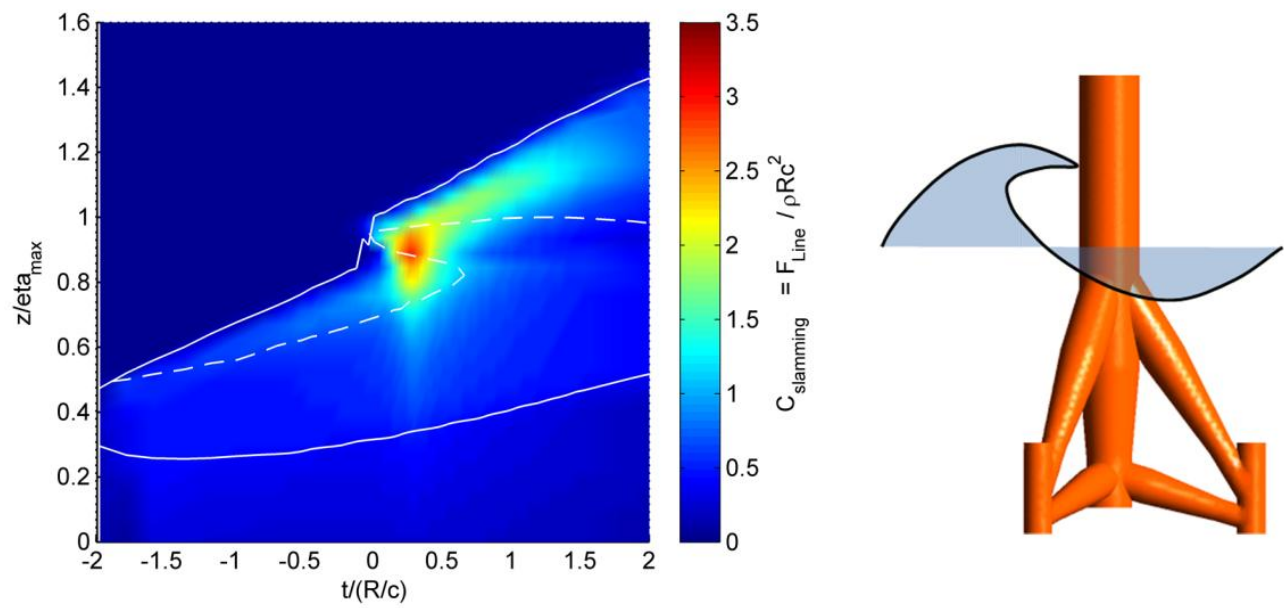

Figure 11. Slamming coefficients of LC 2 over time normalized by time of immergence (radius/wave velocity) and versus relative height " $z /$ eta $_{\max }$ " with water elevations at the cylinder's front (upper solid line) and rear side (lower solid line); the dashed line shows the wave gauge unaffected by the structure.
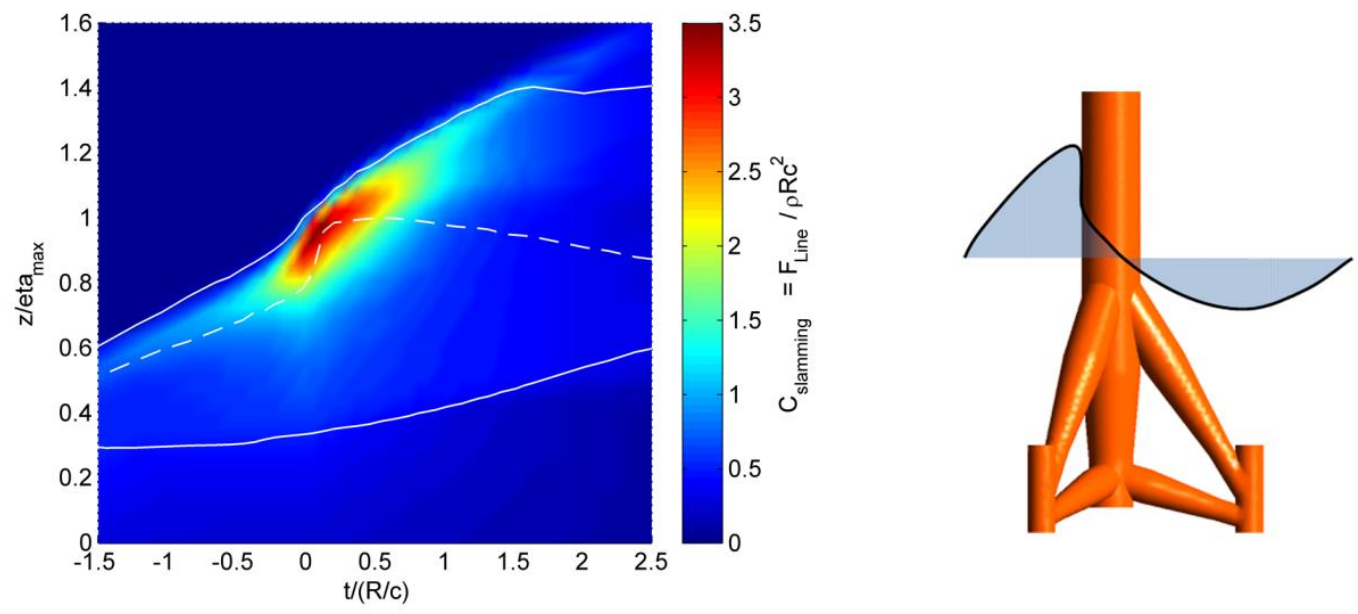

Figure 12. Slamming coefficients of LC 2 over time normalized by time of immergence (radius/wave velocity) and versus relative height " $z /$ eta $_{\max }$ " with water elevations at the cylinder's front (upper solid line) and rear side (lower solid line); the dashed line shows the wave gauge unaffected by the structure. 
on the backside. Figure 13 is equivalent to Figure 11 except the reduced limit of the colorbar to 0.5 for illustration purposes and indicates the absence of the supporting force by the red coefficients. The coefficients drop relatively fast from values $>0.45-0.5$ down to 0.3 or 0.2 below the water level on the rear side. Since the water level decreases with a variable gradient along the circumference of the main column, the section without pressure on the backside is slightly smaller than indicated by the two local water levels. For orientation purposes the dashed lines illustrate the shapes of the breaking waves on a level with the cylinder front and without being influenced by the structure. Load cases two and three have explicit wave fronts in contrast to load case one with the broken wave illustrated by the white $\mathrm{x}$ marks. For all cases the time scales on the abscissas are set to zero at the point in time when the wave front contacts the cylinder front.

Figure 11 shows the slamming coefficients for the curling wave tongue encountering the cylinder at the relative height $z_{/} / \mathrm{eta}_{\max }=0.95-1$ on a level with the highest water elevation $\left(\mathrm{z} / \mathrm{eta} \mathrm{max}_{\max }=1\right)$ and $\mathrm{T}_{\mathrm{i}}$ $=0$. The wave tongue spreads upwards and downwards along the cylinder's span during wave propagation, which is why the free surface is defined by three values until $\mathrm{T}_{\mathrm{i}}$ is approximately 0.6. Then, the rising wave front encounters the curled wave tongue from above and after $T_{i}=0.6$ the wave crest passes the main column without a separated tongue, as indicated by the single dashed line. The maximum slamming coefficient for load case two is $C_{S}=3.0$ at z/eta $\max =0.9$ and lasts from $T_{i}=0.25$ to 0.3 (Figure 11). The impact area induced by the curled wave crest (LC 2) approximately ranges from z/eta $_{\max }=0.7-1.2$ by considering the whole impact time. This observation assumes that slamming characteristics in terms of sudden rise times and high force gradients are indicated by coefficients $\mathrm{C}_{\mathrm{S}}>1$, which exceeds the previously described flow force on the basis of the wave celerity. The simultaneously impacted area along the span (denoted as "curling factor" in literature) reduces to a relative width of $0.2-0.3$, which is centered near $z /$ eta $a_{\max }=0.9$ at the early stages of slamming and shifts upwards to $\mathrm{z} / \mathrm{eta}_{\max }=1.1$ within 1.5 times of $\mathrm{T}_{\mathrm{i}}$ (Figure 11). Horizontal cross-sections through the coefficients represent time dependent forces at a specific relative height. Vertical cross-sections show the distribution of the line force along the main column for an arbitrary point in time. The vertical distribution of forces between $T_{i}=0$ and 1.5 hardly shows sections with constant coefficients for load case two. Observations around the maximum value reveal decreasing loads along the cylinder span from 3.0 to 2.0 within roughly $10-15 \%$ of the maximum water elevation. Similar results are found for vertical distributions after the maximum value and underline the variability of impact loads in regions $\mathrm{C}_{\mathrm{S}}>1$. A horizontal intersection through the maximum value gives the time dependent characteristics before and after the maximum coefficient. At this level ( $\mathrm{z}_{\mathrm{eta}} \mathrm{atax}_{\max }=0.9$ ) the rising time is about $0.2 \mathrm{~T}_{\mathrm{i}}$ and taken between the points of time with $C_{S}=1$ until $C_{S}=3$, while the decreasing time takes $0.3 T_{i}$ until $\mathrm{C}_{\mathrm{S}}=1$ again. The slamming duration of approximately 0.4 to $0.5 \mathrm{~T}_{\mathrm{i}}$ for $\mathrm{C}_{\mathrm{S}}$ values exceeding 1 is observed at all various relative heights.

The connection of the impact pressures in Figure 9 and the corresponding coefficients with slamming properties in Figure 11 is visible by the correlating width of the curling factor and the range of intensive pressure in plot (g). Furthermore, the observed point in time $0.25 \leq \mathrm{t} / \mathrm{T}_{\mathrm{i}} \leq 0.30$ as well as the relative height $\mathrm{z}_{\text {eta }} \mathrm{max}_{\max }=0.9$ of the maximum slamming coefficient, matches to the pressure distribution for $\mathrm{t} / \mathrm{T}_{\mathrm{i}}=0.25$ in Figure 9. The comparison of the time dependent pressure distribution and the synchronously developing slamming coefficients emphasizes the differences between line forces and impact pressures. Since the line force takes the whole pressure field of the circumference into account, the maximum force must not necessarily occur simultaneous to the maximum impact pressure, but at the instant of time when the integrated pressures up to $30-40$ degrees are maximum.

Figure 12 illustrates the slamming coefficients for load case three with a vertical wave front section of $\approx 0.2$ eta $_{\max }$ at the instant of impact. $\mathrm{T}_{\mathrm{i}}=0$ is located right before the steep inclination of the unaffected wave gauge record and matches with the impinging wave front at the cylinder. Pressures of load case three generally exceed the pressures of load case two at locations up to 50 degrees along the perimeter between $\mathrm{z} / \mathrm{eta}_{\max }=0.8$ and 1.1 . This leads to visibly higher load coefficients in this region whereby the maximum value of $\mathrm{C}_{\mathrm{S}}=3.5$ occurs at $\mathrm{z}$ eta $\mathrm{max}_{\max }=0.9$ to 1.0 after a rising time of approximately $0.1 \mathrm{~T}_{\mathrm{i}}$. In comparison to LC 2 the region of impact loads spreads a bit wider along the cylinder front and ranges between $\mathrm{z} / \mathrm{eta}_{\max }=0.7$ and 1.3. Vertically distributed slamming characteristics are observed along 0.3 broad sections over time, whereby the center shifts from $\mathrm{z} / \mathrm{eta} \mathrm{max}_{\max }$ $=0.85$ towards 1.1 within nearly $1 \mathrm{~T}_{\mathrm{i}}$. Similar to LC 2 the slamming duration lasts about $0.4 \mathrm{~T}_{\mathrm{i}}$ at each relative height and the loads vary along the cylinder's span as well as over time.

Load case one is illustrated in Figure 10 with the same parameters and values as described for the prior load cases, except the undisturbed wave gauge record. In this case the wave front is indicated by 
single $\mathrm{x}$-marks instead of a continuous dashed line due to the turbulent broken wave front. The maximum slamming coefficient amounts to $C_{S}=2.7$ and is the smallest value out of the three presented load cases. Two coefficient peaks are visible in Figure 10 with the second one slightly weaker $\left(\mathrm{C}_{\mathrm{S}}=2\right)$ at the relative height $\mathrm{z}^{-} \mathrm{eta}_{\max }=0.9$ in contrast to the first one at the lower position $\mathrm{z}^{\mathrm{e}} \mathrm{et}_{\max }=0.65$. These characteristics are connected to the broken wave front, which is less impulsive and encounters the cylinder at the lowest relative height due to the air water mixture and the downward falling wave tongue (Figure 4, left). As a consequence the vertical range of impact coefficients greater 1 is narrower than for the other load cases and located between $\mathrm{z}$ eta $\mathrm{max}_{\max }=0.6$ to 1.1 as well as the curling factor < 0.2. Therefore, the rise time of the first peak with $0.25 \mathrm{~T}_{\mathrm{i}}$ and the impact duration of $0.5-1.0 \mathrm{~T}_{\mathrm{i}}$ at a specific relative height are longest.

The force coefficients change in a relatively similar way outside the impact area and decrease down to $\mathrm{C}_{\mathrm{S}} \geq 0.5$ at relative heights above the water level at the rear side of the cylinder indicated by the lower solid white lines. Shortly below this water level at $\mathrm{z} / \mathrm{eta} \mathrm{m}_{\max } \approx 0.2$ up to 0.4 the coefficients drop to values around $C_{S}=0.25-0.3$ in all three load cases as exemplarily illustrated in Figure 13 . The velocities at these relative heights range between $2.0-2.4 \mathrm{~m} / \mathrm{s}$ according to Figure 8 and remain relatively long in contrast to the high variation near the wave crest. The $C_{S}$ coefficients in this region match the given $C_{D}$ values by Schlichting (1982) for a cylinder in steady flow, which leads to the assumption that the flow conditions for short periods of time might be regarded as "quasi-static" or stationary. In Figure 13 this would be the case at relative heights where only minor changes in color and magnitude occur in horizontal direction. The distance along the abscissa gives the period of time for the stationary flow condition, e.g. 2 times $T_{i}$ between $z /$ eta $a_{\max }=0-0.2$. Vice versa, regions with varying coefficients over time indicate unsteady flow conditions and outer boundaries of impact loads.

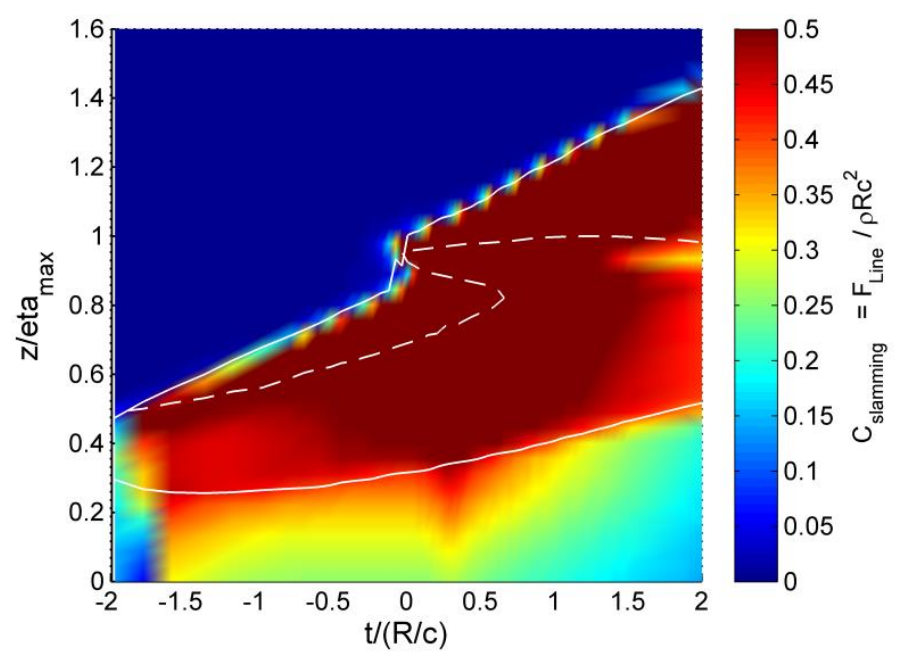

Figure 13. Slamming coefficients of LC 2 analogous to Figure 10 with emphasized coefficients of lower magnitude to classify between potential regions of slamming and streaming forces.

\section{SUMMARY AND CONCLUSION}

This study is motivated by the growing demand for offshore wind energy solutions by means of efficient designs for mass production. An efficient design requires a detailed load assessment, which is why large scale tests and numerical simulations were performed to reveal further insights on kinematics, as well as on pressures and forces in the region of wave impact.

The horizontal water velocities at the onset of wave breaking amount 4.2 to $4.8 \mathrm{~m} / \mathrm{s}$ at the upper $25 \%$ of the positive maximum water elevation, continued by roughly $2.4 \mathrm{~m} / \mathrm{s}$ along almost $50 \%$. of eta $a_{\max }$. The horizontal velocities below the still water level range from 1 to $2 \mathrm{~m} / \mathrm{s}$ with small velocity gradients.

Table 1 gives an overview about the main observed characteristics for the impact of the broken wave (LC 1), the curled wave front (LC 2), and the partly vertical wave front (LC 3). The rising time of the peak coefficients and the impact duration at various relative heights become shorter with decreasing distances of the wave breaking to the tripod, since load case three is the most impulsive breaking type followed by load case two and the broken wave in load case one. Maximum slamming coefficients, 
vertical positions of impact, as well as the curling factors increase with decreasing distances of the wave breaking to the tripod.

The observed impact loads of load case two $\left(C_{S}=3.0\right)$ and three $\left(C_{S}=3.5\right)$ rather match the theoretical slamming coefficient $C_{S}=\pi$ given by von Karman (1929) and adopted by Goda et al. (1966) than the theoretical predictions according to Wienke \& Oumeraci (2005). Last mentioned formulation includes a maximum value of $\mathrm{C}_{S}=2 \pi$ for the partly vertical wave front and is implemented to guidelines for recommended practice. This offers potential for optimization with regard to efficient OWEC designs, since $2 \pi$ is nearly two times higher than $C_{S}=3.5$ observed in this study. In addition, the slamming coefficient $\mathrm{C}_{S}=2 \pi$ is constantly distributed at the cylinder along the section of the upright wave front (referred as curling factor), while the vertical distribution considerably decreases above and below the peak coefficients like a triangular shape along the cylinder's span, seen in Figure 10 to Figure 12. It should be noted that this study does not include full plunging breaking with large vertical wave fronts like the studies of Wienke \& Oumeraci (2005) do, and the direct comparison to load case three is not valid to the full extend. However, the observed results from the physical and numerical model show several differences and reduced loads to estimations according to guidelines. Further aspects on load calculations targeted for efficient OWEC designs including the approach of Goda et al. (1966) are discussed in Hildebrandt \& Schlurmann (2012).

Hanssen and Tørum (1999) state that the Morison (1950) equation applies for load calculations shortly after the cylinder is submerged. This is also observed for load cases two and three in this study and seen in Figure 11 and Figure 12 by the rapidly decreasing $C_{S}$ values to $0.7-0.5$, which is in the range of commonly used $C_{D}$ coefficients for the Morison approach. Additional analysis on the hydrodynamics during impact as well as the relation of impact and quasi-static loads is subject of further analysis.

Table 1. Properties of slamming coefficients and regions of impact for load case (LC) one, two, and three.

\begin{tabular}{|c|c|c|c|c|c|c|}
\hline & \multicolumn{3}{|c|}{ C $_{\text {Slamming }}$ = line force $/ \rho \mathrm{RC}^{2}$} & \multicolumn{3}{c|}{ Region of Impact } \\
\cline { 2 - 7 } & $\begin{array}{c}\text { Max } \\
\text { Value }\end{array}$ & $\begin{array}{c}\text { Position } \\
\text { z/eta }_{\max }\end{array}$ & $\begin{array}{c}\text { Rise } \\
\text { Time }\end{array}$ & $\begin{array}{c}\text { Vertical } \\
\text { Range }\end{array}$ & $\begin{array}{c}\text { Curling } \\
\text { factor }\end{array}$ & $\begin{array}{c}\text { Duration } \\
\text { at } z / \text { eta } \\
\text { max }\end{array}$ \\
\hline LC 1 & $\approx 2.7$ & $\approx 0.65 ; 0.9$ & $\approx 0.25$ & $\approx 0.6-1.1$ & $\approx 0.2$ & $\approx 0.5-1.0$ \\
\hline LC 2 & $\approx 3.0$ & $\approx 0.95$ & $\approx 0.2$ & $\approx 0.7-1.2$ & $\approx 0.2-0.3$ & $\approx 0.4-0.5$ \\
\hline LC 3 & $\approx 3.5$ & $\approx 0.9-1.0$ & $\approx 0.1$ & $\approx 0.7-1.3$ & $\approx 0.3$ & $\approx 0.4$ \\
\hline
\end{tabular}

\section{ACKNOWLEDGMENTS}

The authors gratefully acknowledge the support of the German Federal Environment Ministry (BMU) within the funded project "Ganzheitliches Dimensionierungskonzept für OWEATragstrukturen hinsichtlich Lasten, Langlebigkeit, Gründung und Gesamtstruktur (GIGAWIND alpha ventus - LUH)" (BMU code 0325032/A). Project details and cooperation partners can be collected from www.gigawind.de.

\section{REFERENCES}

Chan, E.-S., Cheong, H.-F., Tan, B.-C., 1995. Laboratory study of plunging wave impacts on vertical cylinders. Coastal Engineering 25 (1-2), 87-107.

Goda, Y., Haranaka, S., Kitahata, M., 1966. Study on impulsive breaking wave forces on piles, Report Port and Harbour Technical Research Institute, 6 (5), 1-30.

Hanssen, A.G., Tørum, A., 1999. Breaking wave forces on tripod concrete structure on shoal. Journal of Waterway, Port, Coastal, and Ocean Engineering, 125 (6), pp 304-310.

Hildebrandt, A., Schlurmann, T., 2012. Breaking wave impact on offshore tripod structures Comparison of large scale experiments, CFD simulations, and DIN recommended practice, Bautechnik, 89. Jahrgang, Heft 5, Berlin: Ernst \& Sohn, (in German).

von Karman, T., 1929. The impact on seaplane floats during landing. National Advisory Committee for Aeronautics. Technical Note No. 321.

Morison, J., O'Brien, M., Johnson, J., Schaaf, S., 1950. The force exerted by surface waves on piles. Petroleum Transactions, AIME 189.

REN21, 2011. Renewables 2011 Global Status Report. REN21 Secretariat, Paris. 
Schlichting, H. (1982). Grenzschicht-Theorie (Boundary-layer theory), Verlag G. Braun, Karlsruhe, 1982 (8. Auflage)

Stahlmann, A., Schlurmann, T., 2012. Investigations on Scour Development at Tripod Foundations for Offshore Wind Turbines: Modeling and Application. Proceedings of the 33rd International Conference on Coastal Engineering - ICCE, Santander, Spain, 2012

Wienke, J., Oumeraci, H., 2005. Breaking wave impact force on a vertical and inclined slender pile theoretical and large-scale model investigations. Coastal Engineering 52 (5), 435-462. 